\title{
Strategic Decision Models Cross-Validation by Use of Decision Reports Information Extraction
}

\author{
L. Hancu
}

\author{
Lucian Hancu \\ 1. "Babes-Bolyai" University of Cluj-Napoca \\ Romania, Cluj-Napoca, 1 M. Kogalniceanu, and \\ 2. SoftProEuro Ltd. Cluj-Napoca \\ Romania, 400614 Cluj-Napoca, 1 Lacul Rosu \\ E-mail: lhancu@softproeuro.ro
}

\begin{abstract}
:
From all the events in the life of a business entity, the Mergers and Acquisitions transactions are one of the most challenging ones, as they drastically affect the life of the involved entities, but also their business stakeholders (like clients or suppliers). The Merger transaction can be seen as a growth crisis in the life of the buyer entity and a strive for survival in the life of the acquired company. Studying such transactions are being a constant preoccupation for both academia and practitioners, modeling mergers in order to predict them - one of the most ambitious task. In this paper, we present our technique of cross-validating the results of our model and use several boosting methods for improving the computed decisions scores.

Keywords: Mergers and Acquisitions, Quantitative Models, Cross-Verification, Boosting Algorithm, Growth Crisis, Business Survival
\end{abstract}

\section{Introduction}

The strategic decisions of the type Mergers and Acquisitions are of crucial importance for the life of both the entities involved in such a process and their stakeholders ones (clients, suppliers, or even competitors). Predicting such transactions are, thus, of great importance for the participants to the economic activities, as the changes in the market conditions can drastically affect the entities, especially the small competitors.

Bearing this in mind, we have previously built a model of predicting future mergers and acquisitions based on the financial statements of the entities involved in such a strategic process and on the correlations between the two entities activity's codes (the so-called Business Dependencies Map [4]). In addition, data regarding previously completed acquisitions are available on the Web and can be easily downloaded and analyzed. Information Extraction from such data can be of great help in cross-verifying the quantitative models for Mergers and Acquisitions.

In this paper, we apply a cross-validation mechanism in order to correlate data manually extracted from the Competitors Council merger decisions reports from 2003 up to 2008 of the type Buyer (the entity who bought another entity) - Target (the entity who was bought) - Seller (the entity who sold its ownership of the Target entity to the Buyer entity) with the results of the MAVOC (Mergers, Acquisitions, Virtualizations or Conservations) quantitative model. The cross-validation occurs only when the Buyer and Target are both Romanian entities and their financial statements are present in the previously-computed database of the Top of the Romanian Entities, so it is possible to compute the MAVOC quantitative score.

Prior to performing such a step, an automatic cleansing is performed on the data extracted from the Competition's Council Decisions Reports, that assures that the entities are found on the databases collecting the financial statement. The cleansing step is crucial as many entities change names after the completion of an acquisition transaction, which makes difficult (or even impossible) the finding of the financial information regarding the specified entity. 
In addition to the cross-validation task, a boosting algorithm is used in order to improve the results of the Mergers and Acquisitions MAVOC model. The boosting algorithm is based on the risk profile of both buyer and the seller and it takes into consideration the risk associated with the two entities activity codes. The boosting algorithm has the scope of improving the acquisition score and downgrading the other scores (especially the inverse acquisition A- and conservation $\mathbf{C}$ ), so as the MAVOC model would output Acquisition for the two companies extracted from the Decision Reports. This boosting technique is required when the acquiring company's financial strength is similar to (or weaker than) the acquired company's financial strength, which would conduct to a false inverse Acquisition (A-) recommendation if used alone without boosting.

The paper is organized as follows. In the following section we provide a brief introduction to the mergers and acquisitions research. This research usually is the result of consultant companies and it takes several years of investigation, when analyzing transactions from several decades. In the subsequent section, we briefly explain our methodology of modeling mergers and the technique of extracting data from decisions reports from the Competition Council, that are later used in cross-validating the model. The fourth section describes our boosting techniques aimed at improving the results of the decision model, whereas the paper ends with a brief summarization of the discussed topics and depicts directions for future work.

\section{Mergers and Acquisitions Transactions}

A thorough analysis of more than 20 years of Italian mergers and acquisitions transactions is done in [7]. The research takes into consideration the interval between 1998 and 2010, in which the authors analyze transactions from various sectors including banking and public services like electricity and gas. It is analyzed the context of such transactions - the Italian economy in the analyzed period, in which the small and medium entities occupy a large percentage of the total amount of business entities. The context is, to some extent, similar to the one of the Romanian market: some transactions were done as privatizations, between the State agencies and private entities (most of them being foreign entities), others being transactions between foreign entities (one which previously acquired the company and which is now willing to exit from the investment).

A special role in these transactions are occupied by investment funds, business entities that acquire several percentages of companies, develop a new business, then sell the company to a third-party investor. While transactions between two companies (competitors, clients or suppliers) are easier to be analyzed - as one could extract features like financial indicators, position on the market, coverage of the market, the case of private funds (also known as investment funds or equity funds) remain a distinct subject of research and it shall be left behind during our research.

The main motives of mergers transactions are depicted in [2]: to affect more rapid growth, gain economies of scale, increase market percentage, expand in the territory, increase stock market value, expand or improve the mix of products, spread risk through diversification, enhance the power and influence of the entity, invest the entity's idle capital, acquire technical knowledge and expertise, counter cyclical of seasonal revenues, obtain managerial talent, gain from tax advantages, obtain more control over the supply sources and/or the retail outlets or to defend against a possible takeover.

Some of these motives are summarized also in [1]. The main aim of the Merger transaction is to realize value, by managing risk and exercising power. The mergers transactions from the market power perspective are also analyzed in [3] and [8]. The former research states that markets are passing through several stages in their way to consolidation, when having almost $90 \%$ of the market power concentrated into the industry giants. The latter research, instead, focuses on 
exploting niche markets for mergers transactions.

The research literature of Mergers and Acquisitions coming from both practitioners but especially from academic researchers is crucial in deriving the criteria for future modeling of mergers and acquisitions transactions. By analyzing the research literature, we have figured out that several mergers motives can be modeled by quantitative variables, while others (denoting especially human resource-related questions) focus on more qualitative results. In this article, we shall concentrate on modeling mergers through quantitative models and improve them by crossvalidations with data previously extracted from the decisions reports of the National Competition Council, that are published through their public Web Information Systems.

\section{Gathering Decision Reports and Cross-Verification of Merger Model}

Modeling mergers decisions, based on the financial indicators of the analyzed entities and the business dependencies of the various business activities (which constitutes the Business Dependency Map [4], has been first explained in [5]. The Mergers Acquisitions Virtualizations or Conservations MAVOC model consists in extracting the financial indicators of the involved entities from the available public Web sources (like the Ministry of Finance or Registry of Commerce).

The model makes use of the two-business entities activity codes, number of employees, financial resources (turnover, tangible assets, intangible assets), market share - whether the entities are part of the local (or national) top of the entities corresponding to the county of each business entity. According to the relations between the financial indicators of the two business entities, a score for each alternative (MAVOC) is computed, the score which is higher ranked is returned as the suggested alternative for the two business entities for the specified year.

One method of verifying the quantitative mergers model would be to extract data from the decision reports published by the Competition Council and cross-verifying the results of the model with the data extracted from these decision reports. In the following paragraph, we briefly explain the methodology we use in extracting data from these reports.

\subsection{Extracting Information from Decision Reports}

Strategic decisions like mergers or takeovers are analyzed by the country's Competition Council (in the case that the two entities are from the same country) or by each country's specific Competition Council (in the case that the two business entities are coming from two different countries). Upon each analysis, a decision report is issued (and usually available using Web Information Systems) which describes the details of the transaction and the Council's acceptance or rejection.

The technique of extracting information from these reports has been thoroughly explained in [6]. We extract information from these reports then cross verify the extracted data with our data sources, in order to obtain relationships of the type Buyer (the entity which bought another entity) - Target (the entity who was bought) - Seller (the entity which previously owned the Target). In some cases, the relationship is restricted to only Buyer - Target, as there is no entity which owns the Target company.

\subsection{Cross-Verification of the model with previously-extracted data}

The extraction of the data resulted in 740 relationships of the form Buyer - Target - Seller, from which $20,27 \%$ of them were business entities coming from Romania. In order to further 
perform cross-validation tasks, we had to check that these entities are business entities (and not public agencies owned by the state), so that we can obtain the financial indicators from the external information sources.

The results of the cross-validation baseline experiment are depicted in the first row of the Table 1 and in the Figure 1. The table synthesizes the percents of the final results of the model for the input coming from the decisions reports: 2,67\% were classified as Mergers, $14 \%$ were classified as inverse acquisitions (if $\mathrm{A}$ and $\mathrm{B}$ are the entities, we denote direct acquisition or $\mathbf{A}+$ the acquisition of $\mathrm{B}$ made by $\mathrm{A}$ and inverse acquisition or $\mathrm{A}$ - the acquisition of $\mathrm{A}$ made by $\mathrm{B}$ ).

In addition, 26, $67 \%$ were classified as Virtualizations (creation of short-term virtual entities for profiting from a business momentum), 30\% as direct acquisitions and $26,67 \%$ as errors. The errors are due to the fact that several values are missing in our Information Systems which contain financial data on business entities. Instead of adding an auto-updater tool to our Information Systems for correcting these errors, we have performed several boosting strategies in order to correct or limit these errors, strategies that are explained in the next section.

The last two columns of the Table 1 represent the relative number of correct answers with respect to all the output of the model including the errors, and the relative number of those answers with respect to the output excluding the errors. The former is computed by dividing the $\mathrm{M}, \mathrm{A}+$ and $\mathrm{V}$ answers to the $\mathrm{M}, \mathrm{A}+, \mathrm{A}-, \mathrm{V}$ and Error answers, while the latter is computed by dividing the $\mathrm{M}, \mathrm{A}+$ and $\mathrm{V}$ answers to the $\mathrm{M}, \mathrm{A}+, \mathrm{A}$ - and $\mathrm{V}$ answers. As previously noted in our experiments, with the introduction of the 250 top of the entities (instead of the previous top of 10 entities in the original model [5]) the number of Conservations $(\mathrm{C})$ results is zero. We consider here the $\mathrm{M}, \mathrm{A}+$ (direct acquisitions) and $\mathrm{V}$ as correct answers for the cross-validations with the decision reports data extraction. During our boosting experiments we shall concentrate in minimizing the number of errors and the number of inverse acquisitions A-.

In the Figure 1 we depict the analytical scores of the model - the maximum between the M, $\mathrm{A}+$ and $\mathrm{V}$ scores in comparison with the A- and $\mathrm{C}$ scores. The image serves at an overview of the baseline experiment. We shall improve the score and present an updated version of the score, during the boosting section.

\section{Boosting Techniques}

Several approaches were required for improving the results of the decisions scores. In this section, we briefly summarize the boosting approaches that we are using.

Experiment 2 Downward model - in order to improve the number of correct answers, we apply a downward strategy to our model: we take the initial year of the transaction and the fiscal IDs of the two entities from the extracted decision reports (see previous paragraph for the methodology). If the model outputs an error, we apply the same model to the previous years from the range 2003..2008 in order to obtain better results (non-error result of the model). The reason for applying this boosting technique is: several transactions completed at the end of a fiscal year are analyzed only in the next year, when one of the entity might not be a valid entity any more, in the mean time being absorbed by the other entity, and, thus, making impossible the gathering of its financial indicators for the current year. The results are depicted in the second row of Table 1 and reveal a slight improvement of the results of the experiments.

Experiments 3 Upward model - a similar mechanism of upward strategy is also used, when the model outputs the same error (indicating that one or both business entities are missing from the Information Systems business data), with the sole difference that we analyze the years after the transaction and output the model decision score, when available. 


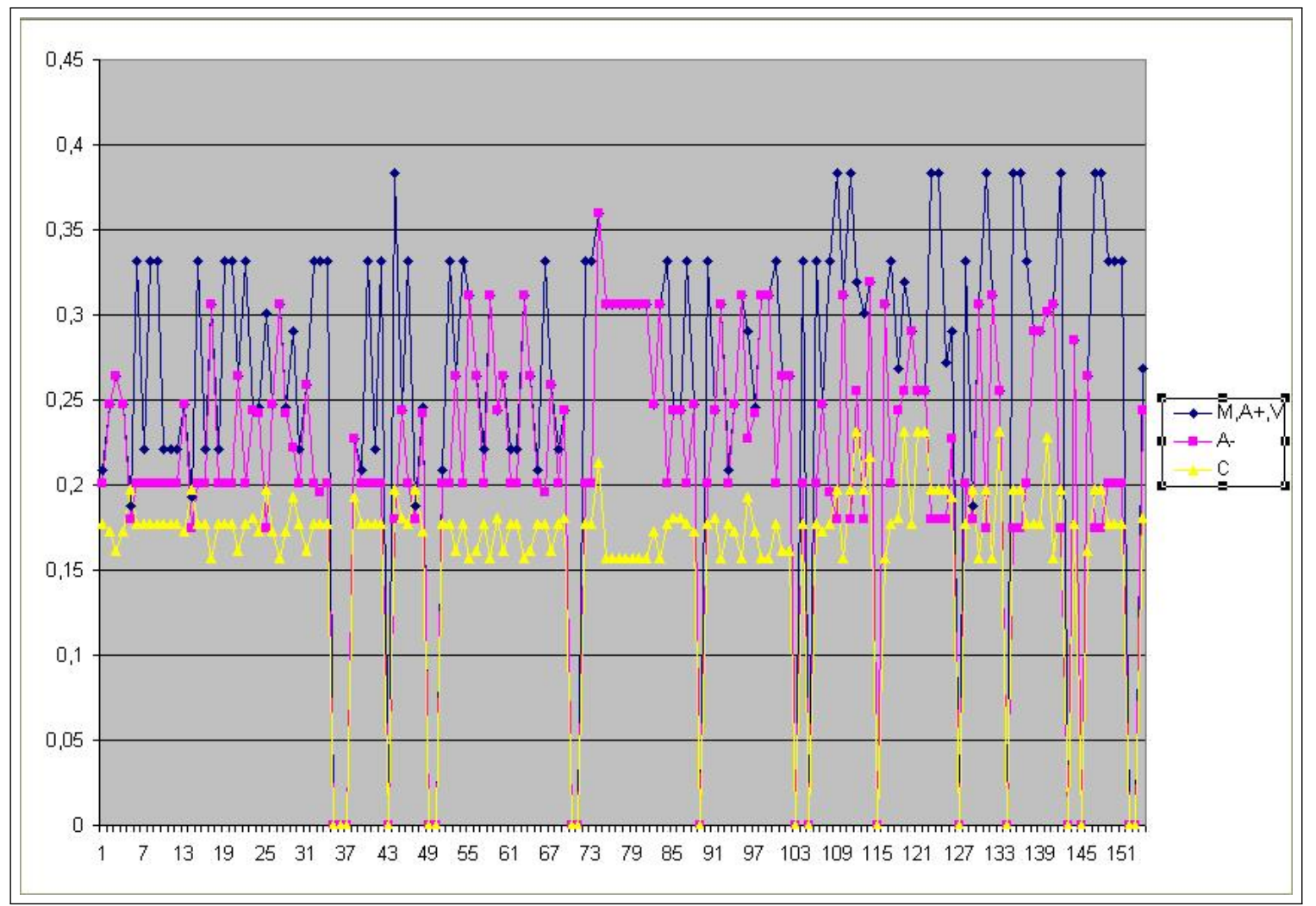

Figure 1: Model score before boosting

Experiments 4 and 5 Using Total incomes instead of Turnover into the model. By manually inspecting the false negative results from our experiments, we have discovered that a number of entities had much higher total incomes with respect to the turnover, which suggested us to use the other indicator into the model in order to improve the results. The difference between the two experiments is a small change in the importance of the Business Classes of the two entities, that affected the results of our experiments; we shall use this strategy in the boosting techniques, discussed below.

Risk-based Boosting: Experiments 6-12 The main idea of this paper was to apply riskbased boosting techniques in order to improve the decision scores of our model. The motivation for this approach relies on the fact that businesses coming from certain business sectors are more willing to accept higher risks than businesses coming from the more conservative business sectors. We have previously developed various techniques for computing the dependency-based risks from the Virtualized Supply Chains; in this article we shall limit at applying boosting techniques to the high risk-tolerant business classes, without insisting in the classification low-medium-high risk Business Class. Thus, higher risks from mergers and acquisitions transactions are supposed to be accepted for the high risk-tolerant business classes.

The Risk-Based Boosting techniques are summarized below:

Model's parameters When the Business Class of the A company is one of the high risktolerant business classes, we use different thresholds for the parameters $K_{1}, K_{2}, K_{3}, K_{4}$ (in the relations 1, 2, 3, 4, as follows: for the first relation we use the lower-bound parameter for higher risk business classes, allowing that weaker business entities acquire stronger ones 


\begin{tabular}{|r|c|c|c|c|c|c|c|}
\hline & $\mathbf{M}$ & A- & V & A+ & Err & Proc $_{1}$ & Proc $_{2}$ \\
\hline 1 & 2,67 & 14 & 26,67 & 30 & 26,67 & 59,34 & 80,91 \\
2 & 2,67 & 16 & 30,67 & 36 & 14,67 & 69,34 & 81,25 \\
3 & 2,6 & 14,94 & 31,17 & 39,61 & 11,69 & 73,38 & 83,08 \\
4 & 2,6 & 16,23 & 30,52 & 38,96 & 11,69 & 72,08 & 81,62 \\
5 & 2,6 & 12,34 & 31,82 & 41,56 & 11,69 & 75,98 & 86,03 \\
6 & 1,3 & 12,99 & 31,17 & 42,86 & 11,69 & 75,33 & 85,29 \\
7 & 5,23 & 13,07 & 26,8 & 43,14 & 11,76 & 75,17 & 85,19 \\
8 & 4,55 & 12,34 & 26,62 & 44,81 & 11,69 & 75,98 & 86,03 \\
9 & 1,3 & 10,39 & 37,01 & 39,61 & 11,69 & 77,92 & 88,23 \\
10 & 12,34 & 7,14 & 29,22 & 39,61 & 11,69 & 81,17 & 91,91 \\
11 & 7,14 & 7,14 & 29,22 & 44,81 & 11,69 & 81,17 & 91,91 \\
12 & 2,6 & 5,19 & 29,87 & 50,65 & 11,69 & $\mathbf{8 3 , 1 2}$ & $\mathbf{9 4 , 1 2}$ \\
\hline
\end{tabular}

Table 1: Boosting experiments

(but within the specified threshold between the intangible assets $A C_{A}$ and the sum between tangible and intangible assets $A I M_{B}$ and $A C_{B}$. The relation 2 specifies that the lowest parameter should be use in less risk-tolerant business classes, whereas the highest should be used in the higher risk-tolerant ones. We point out that the more risk-tolerant business class refers to the business class of A and not to the risk class of B. Similar relations are 3 and 4 when we use the number of employees. More risk-tolerant business classes are those that are willing to assume a higher risk during the acquisition transaction. This could be contracting a credit for the acquisition of the company, or having a weaker company willing to acquire a stronger one for gaining market share.

$$
\begin{gathered}
A C_{A}>K_{1} *\left(A I M_{B}+A C_{B}\right), K_{1} \in\{0.5,1.0\} \\
A C_{B}>K_{2} *\left(A I M_{A}+A C_{A}\right), K_{2} \in\{1.0,2.0\} \\
E M_{A}>K_{3} * E M_{B}, K_{3} \in\{0.5,2.0\} \\
E M_{B}>K_{4} * E M_{A}, K_{4} \in\{2.0,4.0\}
\end{gathered}
$$

Importance of criteria The criteria used in our MAVOC model can be divided into two categories: risk-dependent ones and non-risk-dependent ones. For the risk-dependent ones like the financial criteria, human resources criteria and business classes criterion we use different importance weights than in the standard risk classes. This assures that the risk-dependent variables are more weighted in constructing the final decision score.

Weights of alternatives When using the risk-dependent criteria we provide various weights for the 5 alternatives, that are tuned during the boosting Experiments 6-12. Higher weights are given to the alternatives that express a direct acquisition, whereas gradually lower weights are given to the ones expressing inverse acquisition A-. 


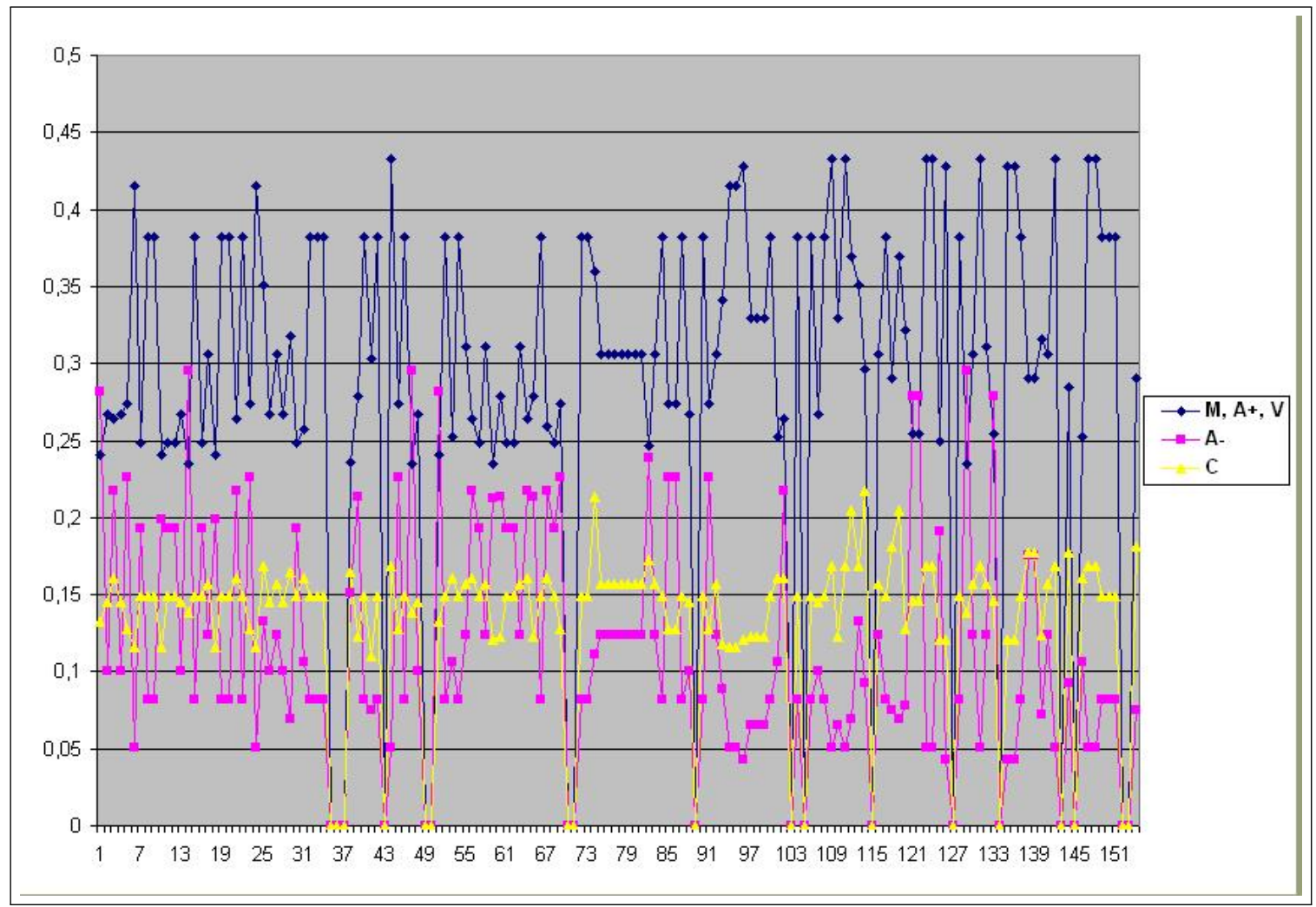

Figure 2: Model score after boosting

The results from our experiments are summarized in Table 1 and Figure 2. The figure shows that the cross-validated results are better than the initial ones. The reason for summing the results of the scores $\mathbf{M}, \mathbf{A}+, \mathbf{V}$ is that we can consider the Merger and Virtualization score as similar to the acquisition score. As stated in the experiments from [5], the Merger score is given to almost equal in financial and human strength of business entities with similar activity codes, whereas the Virtualization score is given to the same kind of business entities, but having different and correlated activity codes.

\section{Conclusions and Future Works}

In this paper we have presented a technique for boosting the results of the merger scores obtained by cross-validating the MAVOC model decision scores when the business classes are ones from more risk-tolerant business classes.

Several factors limit the results of our experiments: the relatively high number of transactions in which one or two of the businesses from the Buyer-Target-Seller relationship are foreign entities (or local businesses hidden behind foreign offshore companies). In these cases, it was not possible to apply the decision score and boost its results. In our future works, we plan to also investigate the possibility to include foreign companies into the model, by enhancing the Information Systems database which contains the financial statements of the analyzed business entities. 


\section{Acknowledgment}

This work was partially supported by the CNCS-UEFISCDI under the grant PN II-RUPD-397/2010. We would like to thank the Combinations of companies M.Sc. students and their academic mentors from the Faculty of Economic Sciences and Business Administration for their contribution in manually extracting the Mergers and Acquisitions informations from the Decisions Reports.

\section{Bibliography}

[1] D. Angwin, Mergers and Acquisitions, Blackwell Publishing, 2007, pg. 21.

[2] H. K. Baker, T. O. Miller, B. J. Ramsperger, A Typology of Mergers Motives, in Akron Business and Law Review, 12(4), 1981, 24-29, reprinted in J. A. Krug, Mergers and Acquisitions, SAGE, 2008, pp. 67-76.

[3] G.K. Deans, F. Kroeger, S. Zeisel, Winning the Merger Endgame, A playbook for Profiting from Industry Consolidation, A.T. Kearney, 2003, pp. 22-95.

[4] L. Hancu, Data-Mining Techniques for Supporting Merging Decisions, in International Journal of Computers, Communications and Control, Suppl. Issue, 2008, pp. 322-326.

[5] L. Hancu, Pruning Decision Trees for Easing Complex Strategic Decisions, in Annals of the Tiberiu Popoviciu Seminar, Volume 6, 2008, pp. 194-203.

[6] L. Hancu, Mining Strategic Decisions Information Systems for Predicting Future Market Concentrations, in Proceedings of the International IADIS Information Systems Conference, March 2012, Berlin, Germany.

[7] KPMG, 20 anni di MESA - Fusioni e acquisizioni in Italia dal 1988 al 2010 (20 years of M\&As - Mergers and Acquisitions in Italy from 1988 to 2010), EGEA, 2010.

[8] F. Kroeger, A. Vizjak, M. Moriarty, Beating the Global Consolidation Endgame, A. T. Kearney, 2008. 